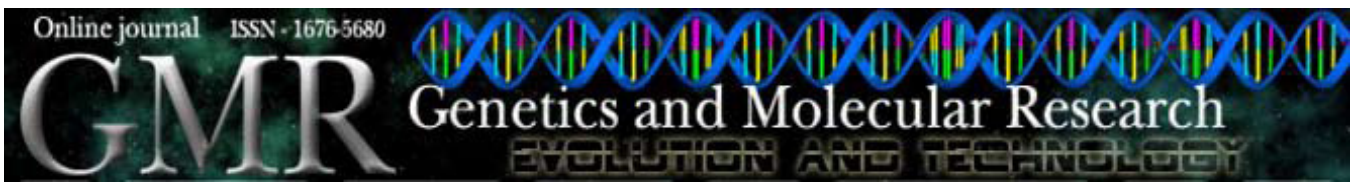

Thesis Abstract

\title{
Comparative study of the cytogenetic and molecular characteristics of Triatoma maculata and T. pseudomaculata (Heteroptera, Triatominae)
}

\author{
P.P. Mendonça
}

2010. Pós-graduação em Genética, Instituto de Biociências, Letras e Ciências Exatas, UNESP/IBILCE, São José do Rio Preto, SP, Brasil. Master's thesis. Orienting Prof:: Dr. Maria Tercília Vilela de Azeredo-Oliveira.

Triatomines are hematophagous insects of great concern in public health because they are vectors of Trypanosoma cruzi, a protozoan that causes Chagas disease. Triatomines are also of great genetic interest, because they possess holocentric chromosomes and undergo an unusual form of meiosis with post-reductional segregation of sex chromosomes. Recent studies based on molecular markers have aimed to understand the evolutionary history of triatomines. Ribosomal DNA (rDNA) analyses are frequently used to elucidate the evolution of a given species, which can help to infer evolutionary relationships between species. Individual copies of rDNA do not accumulate mutations independently because they belong to multigene families, resulting in slight intraspecific and important interspecific variation. In this study, a comparative analysis was performed between the species Triatoma maculata and T. pseudomaculata, based on cytogenetic techniques of lacto-acetic orcein staining, silver impregnation, C-banding, Feulgen reaction, and CMA/DAPI C-banding. We also compared the species by sequencing the rDNA internal transcribed spacer region 1 (ITS-1) in order to determine the degree of homology between the two species. The karyograms of the two species revealed ten autosomes and one pair of sexual chromosomes $(2 n=22)$. In the meiotic cycle, nucleolar fragmentation was observed during the final stages of meiotic prophase I. Nucleolar bodies were found in some meiotic metaphases of $T$. pseudomaculata, which is evidence of nucleolar persistence. C-banding revealed that the Y chromosome is heterochromatic in both species. The ITS- 1 rDNA sequences indicated that the species showed a close proximity to each other and a high degree of homology $(98.5 \%)$. The knowledge obtained in this study contributes to the understanding of the interrelationship and distribution of these species, and offers new tools that can aid in the prevention of Chagas disease.

Key words: Triatomines; Holocentric chromosomes; Spermatogenesis;

Nucleolar organizer region; Heterochromatin; Ribosomal DNA 